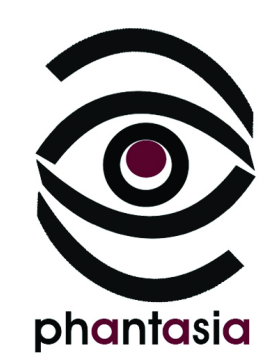

\title{
La vérité aux limites du discours : la « performance » politique des cyniques
}

\author{
Arianna Sforzini \\ LIS, Université Paris-Est Créteil
}

\section{Introduction. Dire-vrai, faire-vrai : la parrêsia cynique}

Dans un entretien de 1984, «Le souci de la vérité », Foucault affirme que ce qu'il a essayé de faire dans ses analyses, «c'est l'histoire des rapports que la pensée entretient avec la vérité ; l'histoire de la pensée en tant qu'elle est pensée de la vérité. Tous ceux qui disent que pour moi la vérité n'existe pas sont des esprits simplistes $»^{1}$. Faut-il donner fois à ces affirmations tardives de la part de Foucault? Il a y sans doute des effets de réélaboration dans le regard rétrospectif qu'il porte sur ses travaux antérieurs. L'idée même d'un « bilan » pour ce qui concerne la pensée foucaldienne est d'ailleurs une opération dangereuse, car on risque de perdre de vue la spécificité de chaque recherche et l'ampleur des déplacements successifs. On risque de se trouver piégé par un désir de système qui est précisément ce contre quoi Foucault n'a cessé de faire jouer sa propre pensée. Cela dit, la question de la vérité, sous l'angle de son histoire politique, traverse effectivement, comme le veut Foucault dans les années quatre-vingt, sa pratique de philosophe. Foucault construit des archéologies et des généalogies de la vérité qui permettent de retrouver dans les plis des histoires des pratiques de vérité des formes de dire-vrai différentes, inattendues, subversives, produisant un effet d'écart et de mise en question de la Vérité de la tradition scientifique et philosophique occidentale. La parrêsia, le dire-vrai libre et courageux, dans le monde antique est sans aucun doute une vérité de ce genre, une vérité résistante dont la formulation ne vise pas à représenter la réalité du monde mais à changer la modalité d'être et d'existence de celui qui l'affirme ainsi que de celui qui l'écoute. Mais cette question de la vérité comme «altération », selon la célèbre affirmation du dernier cours de Foucault, Le courage de la vérité $e^{2}$, traverse en réalité le parcours foucaldien, comme exigence de dessiner les traits d'un nouveau rapport au vrai qui ne soit pas de simple restitution/concordance mais de transformation et de lutte.

Dans le présent article, nous souhaiterions réfléchir à un paradoxe inhérent à la préoccupation foucaldienne de parcourir la généalogie de vérités autres et altérantes. Ce paradoxe touche directement la question du langage et notamment de la mise en parole et en discours de la vérité : bien que Foucault continue explicitement, jusqu'à ses derniers cours au Collège de France, à se situer dans le cadre d'une histoire du «discours vrai », du «dire-vrai», cette forme de parole de vérité prend de plus en plus, en particulier avec l'analyse de la parrêsia cynique, la forme d'un "faire-vrai », d'une attitude, d'une performance des corps qui, à la limite, peut aussi se passer du rapport au discours énoncé pour ne laisser signifier que les gestes et l'existence corporelle. Nous explorerons ce paradoxe à travers le rapport de la question de la vérité à deux concepts fondamentaux dans la pratique cynique : le corps et la nature. Nous ouvrirons ensuite un rapprochement entre la pratique philosophique foucaldienne et la question du performatif et de la performance, en nous demandant si les travaux foucaldiens sur la vérité ne gagneraient

\footnotetext{
${ }^{1}$ Foucault M., «Le souci de la vérité », in Dits et écrits II. 1976-1988, texte n 350, Paris, Gallimard, «Quarto », 2001, p. 1488.

${ }^{2}$ «Il n'y a pas d'instauration de la vérité sans une position essentielle de l'altérité ; la vérité, ce n'est jamais le même »; Foucault M., Le courage de la vérité. Le gouvernement de soi et des autres II. Cours au Collège de France. 19831984, F. Gros éd., Paris, Seuil/Gallimard, 2009, p. 311.
} 
pas à être relus comme des performances esthétiques et politiques de la vérité. Quelle portée peut-on donner au dire-vrai, lorsque les mots s'estompent pour ne laisser parler qu'un noyau corporel et matériel, « animal », de vérité?

\section{Quand la vérité se fait corps}

Commençons par la question du corps, au cœur du paradoxe d'un dire-vrai cynique aux limites mêmes du discursif. Les analyses de la parrêsia cynique constituent un moment fameux et très commenté du dernier cours foucaldien au Collège de France (Le courage de la vérité, cf. notamment la leçon du 29 février 1984). Foucault y décrit une manière non seulement de dire mais de vivre la vérité qui passe par son incarnation radicale dans des comportements, des gestes, des attitudes du corps, les habits, tous les détails de l'existence corporelle. Le cynique se reconnait d'ailleurs par des référents physiques connus et codifiés dans le monde antique : barbe et cheveux longs, pieds nus, saleté du corps et usure des vêtements-haillons, port d'une besace et d'un bâton, etc. Il ne s'agit pas simplement d'un « masque » à porter pour se travestir en cynique. La dramatisation, la spectacularisation du corps nu et malpropre est un véritable mode d'être qui fait de cette forme d'existence la manifestation (ostensio en latin), l'ostentation de la vérité elle-même. Vivre selon le vrai, cela ne signifie pas pour le cynique rendre sa vie conforme à des logoi appris, selon un mouvement d'incorporation de la vérité comme principe d'action. Le processus va encore plus loin, jusqu'à effacer la différence entre corps, vie et vérité. L'existence corporelle devient le double, au sens artaudien du terme ${ }^{3}$, de la vérité : une vie de vérité et une vérité vivante, dans une confusion créatrice et critique qui décourage toute tentative de définition, identification et distinction. Le cynisme est, dit Foucault, le « corps-même de la vérité [...] rendu visible, dans un certain style de vie. La vie comme présence immédiate, éclatante et sauvage de la vérité » ${ }^{4}$. Le cynique fait en sorte que «la vérité $[\ldots]$ prenne corps dans son corps ${ }^{5}$, que le corps devienne le lieu vivant de la vérité. Les gestes et les attitudes du corps ne sont pas de simples ornements extérieurs, signes d'appartenance à un mouvement doctrinal, mais la condition nécessaire à la vie de vérité, à l'existence philosophique $^{6}$. Le cynique apparaît alors « comme la statue visible de la vérité [...] : l'être même du vrai, rendu visible à travers le corps $»^{7}$. Le corps cynique est le théâtre vivant de la vérité.

Par cette théâtralisation corporelle du vrai dans la vie, le cynisme pousse alors à ses limites la possibilité de penser l'articulation entre le dire-vrai et les pratiques d'existence ${ }^{8}$, jusqu'au point où le langage verbal n'est plus nécessaire et presque un obstacle. Le cynique n'a pas besoin de faire des grands discours, encore moins de donner une forme systématique à sa doctrine (même si Foucault sous-estimait sans doute la portée théorique du mouvement cynique). Il lui suffit d'exposer la manière de vivre son corps selon la vérité, pour faire de cette vérité non plus une lumière qui indique la voie vers le Bien mais la pierre de touche, le moment de contestation de toute vérité et valeur reçues. L'existence cynique devient ainsi, selon la reconstruction de Foucault, le lieu d'une vérité conçue comme instrument, réduction et épreuve. La vie permet au cynique de mettre en scène cette vérité dont son corps et ses comportements doivent être la manifestation. L'incarnation du vrai dans l'existence réduit ensuite toute vérité ou identité conventionnelles à un noyau d'élémentaire : c'est le corps lui-même, sa naturalité la plus immédiate et dérangeante, l'animalité, l'essentialité de l'existence physique la plus dénuée qui conteste les prétentions philosophiques à une idéalité pure. La coïncidence de la vie et de la vérité a donc, troisièmement, une valeur de mise à l'épreuve de la vérité : que reste-t-il de toute vérité, une fois qu'elle a été réduite au scandale de la pure existence corporelle ? Que faire d'une manifestation de la vérité qui ne se limite pas au dire-vrai, aussi dérangeant soit-il, mais à la limite ne parle pas, afin de théâtraliser la parrêsia dans la forme même de l'existence et rendre ainsi son spectacle littéralement insupportable pour les philosophes et idéologues du vrai ?

On touche ici évidemment aux limites de la parrêsia et plus généralement d'un dire-vrai conçu comme élément de lutte et de liberté dans un champ agonistique de relations de pouvoir. Foucault rappelle plusieurs exemples de ce scandale des corps dans les vies des cyniques anciens - Diogène qui se masturbe et défèque

\footnotetext{
${ }^{3}$ Cf. Artaud A., Le théâtre et son double, Paris, Gallimard, 1938.

${ }^{4}$ Foucault M., Le courage de la vérité, op. cit., p. 160.

${ }^{5}$ Ibid., p. 284.

${ }^{6}$ Cf. Goulet-Cazé M.-O., L'ascèse cynique, Paris, Vrin, 1986.

${ }^{7}$ Foucault M., Le courage de la vérité, op. cit., p. 288.

${ }^{8}$ Cf. BRAnHAM B., « Diogenes' Rhetoric and the Invention of Cynicism », in M.-O. Goulet-Cazé et Richard Goulet (dir.), Le Cynisme ancien et ses prolongements. Actes du colloque international du CNRS (Paris, 22-25 juillet 1991, Paris, PUF, 1993.
} 
sur la place publique, qui boit dans les flaques d'eau comme un chien, etc. Peut-on définir ces gestes comme des formes de parrêsia, des affirmations de vérité ? Pour Foucault en tout cas, le corps exposé et dérangeant du cynique qui ne craint pas les yeux d'autrui et prend la pudeur pour de l'hypocrisie, qui vit au grand jour, fait l'amour, mange, se soulage sur la place publique, est une manifestation de vérité plus puissante et plus scandaleuse que tout discours. On peut aussi simplement reprendre l'anecdote concernant Cratès et sa future femme Hipparchia : Hipparchia harcelait Cratès pour qu'il l'épouse, mais ce dernier refusait en considérant le mariage incompatible avec la vie cynique. Face à l'insistance d'Hipparchia, qui menace même de se suicider s'il ne l'épouse pas, Cratès se met enfin nu face à elle et lui dit : "Voilà ton mari, voilà ce qu'il possède, décide-toi parce que tu ne seras pas ma femme si tu partages pas mon mode de vie $»^{9}$. Dans cette histoire d'épousailles paradoxales et grotesques, on voit bien comment la vie devient la manifestation même de la vérité assumée et incarnée par le cynique : une alèthurgie à part entière. Et la question du sens du direvrai dans les gestes véridiques cyniques apparaît dans toute sa complexité : dans cet exemple, l'acte de parrêsia de Cratès réside-t-il dans son discours à Hipparchia ou dans son geste de dépouillement ? Si la mise à nu du corps de Cratès est une forme de parrêsia, quid de la discursivité de la vérité ? Quid de la vérité même, devenue exposition et réduction à l'élémentaire du corps ?

\section{La « naturalité » de la vérité comme instance critique}

La question de la nature est un autre biais pour réfléchir à ce paradoxe d'une parrêsia, d'un dire-vrai qui n'a pas besoin de se déployer discursivement mais prend corps et vie dans l'existence toute entière de celui qui l'accepte et l'incarne. Il est clair que la nature fonctionne dans ce contexte des analyses foucaldiennes sur les pratiques antiques de soi et de vérité, non pas comme une essence cachée à découvrir mais comme un pivot critique. Ce qui est en jeu avec la naturalité de l'existence vraie, c'est une manière de faire jouer le plus matériel et le plus concret de la vie comme un opérateur de transformation. Encore une fois, la vérité (cette fois, de la nature) est à entendre dans un sens qui est simultanément instrumental, réducteur et expérimental, comme c'était le cas pour la coïncidence de la vie et de la vérité. La nature est un instrument de l'affirmation du vrai ; elle permet d'en réduire la portée à son noyau essentiel, se libérant du poids des conventions ; elle devient, plutôt que secret caché d'une identité vraie, le lieu d'une mise à l'épreuve des vérités conventionnellement acceptées. Il faut alors non pas tant redécouvrir une nature cachée mais la faire exister comme le produit d'une série de pratiques critiques et éthiques. Il s'agit, dit Foucault, de « redevenir ce qu'on aurait dû être mais que l'on n'a jamais été $»^{10}$.

Cette notion de nature comme mouvement critique est déjà présent dans le cours au Collège de France de 1982 (dans la leçon du 10 février), et déjà à travers l'exemple d'un penseur cynique : Demetrius. Foucault reprend un texte de ce philosophe cité assez longuement par Sénèque dans le De Beneficiis, livre VII (il s'agit donc du cynisme de l'âge impérial, que l'on connaît par l'intermédiaire d'un philosophe du stoïcisme romain, Sénèque, l'un des auteurs les plus aimés et les plus étudiés et cités par Foucault dans ses derniers travaux). Il s'agit, dans ce passage de Demetrius cité par Sénèque, de définir quelles sont les connaissances utiles et inutiles pour l'existence humaine. On pourrait penser de manière hâtive que le partage se fait au niveau des contenus : les connaissances inutiles relèvent des causes extérieures, de la nature comme univers physique, tandis que les connaissances utiles se focalisent sur la nature humaine, l'essence du logos et de l'âme de l'homme. Or pour Demetrius, selon Foucault, les choses ne se passent absolument pas comme ça. Il ne nous invite pas à une conversion du regard de l'extérieur à l'intérieur, du monde à la nature humaine, de la nature cosmique aux profondeurs de son âme. «Il ne veut pas substituer aux secrets de la nature les secrets de la conscience. Il n'est jamais question que du monde. Il n'est jamais question que des autres. Il n'est jamais question que de ce qui nous entoure. Il s'agit simplement de les savoir autrement $»^{11}$.

Il y a donc un jeu conceptuel et éthique selon Foucault entre les deux dimensions de la nature-monde et de la nature-soi, qui ne sont pas opposées comme deux entités distinctes relevant de deux théories bien spécifiques (le savoir du Monde et le savoir du Soi). Nature-monde et nature-soi indiquent plutôt deux manières diverses de connaître : selon les causes et selon les relations entre soi, autres, monde, kosmos, dieux etc. C'est ce dernier savoir relationnel qui pourra servir comme armature dans le combat de l'existence et qui

\footnotetext{
${ }^{9}$ LAËrCe Diogène, Vies et doctrines ..., trad. M.-O. Goulet-Cazé, VI, § 96, p. 760.

10 Foucault M., L’herméneutique du sujet. Cours au Collège de France. 1981-1982, F. Gros éd., Paris, Seuil/Gallimard, 2001, p. 92.

${ }^{11}$ Ibid., p. 225 ; nous soulignons.
} 
transformera le soi lui-même dans son chemin de construction et de perfectionnement en tant que sujet éthique, sujet d'un êthos, d'un style de vie. La nature entre donc dans le jeu de la construction de soi non pas comme essence de l'âme ou loi universelle reprise du kosmos et appliquée à l'intériorité de la conscience, mais comme un triple déplacement critique : elle est relation, arme et principe de transformation. Elle parle de nous dans notre rapport à ce qui nous entoure, elle nous permet de nous équiper contre les aléas de l'existence et de nous façonner donc en sujets d'une vie éthique : d'une existence nouvelle, libre, stylisée et réfléchie. La nature est donc bien un "critère » de "cette réformation critique, de cette réforme de soi », mais « une nature qui n'a jamais été donnée, n'est jamais apparue comme telle dans l'individu humain, à quelque âge que ce soit (et) prend tout naturellement l'allure d'un décapage par rapport à l'enseignement reçu, par rapport aux habitudes établies et par rapport au milieu » ${ }^{12}$. La nature est une création différentielle de soi.

Revenons au Courage de la vérité, et notamment à la leçon du 14 mars 1984. Foucault reprend le thème de la nature par rapport à l'analyse du cynisme ancien, en le concevant en premier lieu comme une « épreuve de vérité », un exercice à travers la réduction à l'élémentaire et à l'animalité. À nouveau, la nature n'émerge pas comme ce qui est donné à l'existence humaine mais comme ce qui reste à reconquérir dans un effort perpétuel de prise de distance par rapport aux acquis et aux paresses de l'habitude. L'une des conceptions traditionnelles de la vie de vérité, du vrai associé à l'existence (Foucault reprend en particulier les significations d'alêtheia selon Platon), est en effet celle de rectitude : une vie vraie est une "vie droite", juste, conforme aux lois. Or les cyniques ne contestent pas directement cette notion du vrai, ne disent pas une vérité autre, mais ils la mettent en scène, encore une fois, dans leurs corps et leurs vies concrètes. Et en théâtralisant la vérité comme vie droite, en la faisant vivre dans leurs corps, en faisant de leurs existences les doubles de ce vrai, ils en font exploser toutes les contradictions. Il y a en effet une ambiguïté fondamentale dans le concept de «loi». Les cyniques veulent bien suivre les lois, mais pas celles contingentes des hommes et de la vie politique, plutôt les lois invariables de la Nature. Or quelle nature ? La nature dans sa valeur la plus animale, la nature des bêtes. Pour les cyniques mener une vie « droite », « suivre la Nature », c'est vivre comme le font les animaux : avec la maison sur les épaules comme un escargot, en pissant en public sans vergogne comme un chien, en exerçant l'amour libre, l'inceste ou l'anthropophagie. Foucault cite par exemple Dion Chrysostome qui revient sur le mythe d'CEdipe, en considérant que sa réaction face à l'oracle fut bien sotte et excessive. À la Pythie qui lui annonçait qu'il aurait partagé le lit avec sa mère et tué son père, en devenant en même temps père et frère de ses enfants, il aurait dû répondre : "Ma foi ! les coqs ne font pas tant d'histoires pour de telles aventures, non plus que les chiens, ni les ânes " ${ }^{13}$. " Il y avait donc là un modèle naturel qu'CEdipe n'a pas reconnu parce qu'il n'a pas été capable de se connaitre lui-même et de retrouver en lui-même un des noyaux de sa naturalité $\rangle^{14}$.

Foucault souhaite-t-il donc nous inviter tous à pratiquer l'inceste ou l'anthropophagie ? Évidemment non. Il nous invite à lire dans la provocation ce qui la soutient et la pousse au-delà d'elle-même, c'est-à-dire une remise en cause radicale de ce que nous pensons être naturel et par conséquent vrai, juste et droit. Dans les exemplarités animales des cyniques il faut entendre que l'animalité ne constitue pas une donnée immanente à quoi se conformer. Elle est l'élément d'une épreuve, d'un travail éthique (repousser les limites de la fatigue et du courage, s'endurcir). "L'animalité, c'est un exercice. C'est une tâche pour soi-même, et c'est en même temps un scandale pour les autres $»^{15}$. Et encore, dans un passage du manuscrit non prononcé lors du cours, Foucault ajoute que «la vie naturelle du cynique a une fonction maïeutique. Il s'agit pour lui de tenter, d'éprouver toutes les vérités qui peuvent être acceptées et révérées par les hommes " ${ }^{16}$. Dans une note de lecture conservée dans le fonds Foucault de la Bibliothèque nationale de France ${ }^{17}$, Foucault cite Julien, Contre les cyniques ignorants (§ 15), sur la question de l'obscénité ; Diogène

foule aux pieds le fumier de l'orgueil, il se rit de ceux qui cachent dans l'ombre la satisfaction de leurs nécessités naturelles - je veux dire l'expulsion de leurs excréments - mais qui, en plein milieu des places

\footnotetext{
${ }^{12}$ Ibid., p. 92.

13 Chrysostome Dion, Discours X: «Diogène, ou Des domestiques », in Les cyniques grecs. Fragments et témoignages, éd. et trad. fr. L. Paquet, Ottawa, Éd. de l'Université d'Ottawa, 1975 [nouvelle éd. : Paris, Librairie générale française, 1992], p. 313.

${ }_{15}^{14}$ FoucAult M., Le courage de la vérité, op. cit., p. 244.

${ }^{15}$ Ibid., p. 245.

${ }^{16}$ Ibid., p. 244.

${ }^{17}$ Boîte 23, chemise 16, Archives Foucault, Bibliothèque nationale de France, NAF 28730.
} 
publiques et des cités, commettent des actes plus violemment contraire aux exigences de la nature, tels que vols d'argent, calomnies, assignations injustes et poursuite d'autres pratiques aussi immondes.

La nature n'est donc absolument pas quelque chose d'évident pour l'homme ; rien de plus conventionnel que ce que nous croyons être naturel. Au miroir de l'animalité, la nature devient « une étrangeté », « une extériorité » et « une guerre ${ }^{18}$, pour reprendre des termes que Foucault utilise dans le même cours à propos de la vérité. Nous pouvons ainsi revenir de la nature à la question de la vérité et de son langage pour Foucault : que faire d'un dire-vrai qui non seulement ne prétend pas dévoiler l'essence et le logos du monde, mais qui renverse l'ordre entre les termes en jeu et fait d'une nature ouverte, agressive, matériellement et farouchement animale, la seule vérité pour laquelle combattre ? Quelle vérité muette disent les cyniques redevenus animaux, leurs corps et leurs existences modelés sur la naturalité des bêtes ? C'est en réalité la vérité d'un militantisme, d'un engagement politique, le seul que Foucault puisse concevoir en dehors de toute idéologie fédératrice. L'opération cynique n'est en effet pas menée par simple goût de la provocation, il faut le rappeler : elle entend secouer ses contemporains; déclencher des réveils, des prises de conscience, des désirs neufs ; susciter de nouvelles manières de vivre. Les corps cyniques instaurent, dit Foucault, « un militantisme ouvert, universel, agressif, un militantisme dans le monde, contre le monde $\gg{ }^{19}$. Ce militantisme ouvert, il faut l'entendre comme : 1. une lutte potentiellement pour tous et de tous, une bataille que tout le monde peut reprendre à son compte, qui n'a pas besoin d'éducation particulière ou d'un certain statut social ou politique; 2. une lutte adressée sans cesse et sauvagement à toutes les faiblesses et les masques d'hypocrisie d'une société ; 3. une lutte qui veut faire bien plus que rendre l'homme sage : elle veut changer la réalité, mettant la construction de soi et de son corps à l'épreuve du rapport aux autres et au monde.

Voilà ce qu'est la vérité pour Foucault lecteur des cyniques : non pas une règle rationnelle immanente, non pas un idéal universel à adresser à tous, mais un corps et un cri de lutte utilisant sa naturalité comme une arme pour transformer le monde. On comprend mieux pourquoi Foucault a pu parler du cynisme, toujours dans son cours de 1984 , comme d'une «catégorie transhistorique ${ }^{20}$. Cette notion même de "catégorie transhistorique » est très étonnante à première vue et paraît contredire tout ce que la pensée de Foucault a essayé d'affirmer depuis ses débuts : qu'il n'existe pas d'extériorité par rapport à l'histoire, pas de concepts traversant l'histoire comme des universaux dont le sens garderait des caractères immuables à travers les différentes époques. Foucault touche-t-il avec le cynisme à une sorte de cœur « vrai » et « naturel» de la philosophie comme manière de vivre ? Oui, dans un certain sens, mais à condition de comprendre ces mots de « vrai » et de «naturel » comme les cyniques eux-mêmes le concevaient selon Foucault. La vraie nature est celle qui défie la naturalité qui nous est imposée ; la vérité naturelle celle qui fait de toute vérité, éprouvée dans les corps encore plus que dans les mots, la manifestation d'un rapport qui transforme le réel. L'unique dimension «transcendantale » à l'histoire est celle de la critique, la possibilité d'ouvrir un espace de différence et de rupture ; le seul militantisme dans lequel Foucault se reconnaît est celui qui engage la déformation des conventions de vérité, pour les fragiliser dans leur contingence et éventuellement les modifier. Le «scandale de la vérité », c'est cela qui fait du cynisme une virtualité capable de traverser diagonalement l'histoire. Transhistorique et universel parce que réactivable dans une lutte immanente à l'actualité, le théâtre du vrai incarné par le cynique est alors une manière de repenser aujourd'hui, à travers l'histoire : une résistance efficace sans être essentialiste ; une vérité vécue ne se posant pourtant pas comme le critère absolu du vrai ; une forme d'existence politique qui fait de la vérité une force sans jamais se mettre dans la position de celui qui gouverne et qui impose donc institutionnellement son discours vrai.

\section{La recherche du vrai comme création et performance. Conclusions ouvertes}

Peut-on alors affirmer que la force militante des analyses foucaldiennes du cynisme résout, d'une certaine manière, l'énigme d'une parrêsia cynique paradoxale, où les gestes prennent la place des mots dans un direvrai ostensif et physique ? Il vaut mieux dire que le paradoxe demeure, tout en prenant son sens à l'intérieur de la démarche foucaldienne, de cette tentative-limite de penser une vérité qui ne soit pas simplement à connaître mais à faire et faire agir comme une dynamique de déplacement et de création. La vérité dont la vie est le théâtre est une vérité qui n'a guère besoin et n'a guère recours au discours; comme le dit Foucault

\footnotetext{
${ }^{18}$ Foucault M., Le courage de la vérité, op. cit., p. 214.

${ }^{19}$ Ibid., p. 262.

${ }^{20}$ Ibid., p. 161
} 
«bien peu de vérité est indispensable pour qui veut vivre vraiment, et [...] bien peu de vie est nécessaire quand on tient vraiment à la vérité ${ }^{21}$. Mais c'est précisément là, aux pointes extrêmes de son discours sur le dire-vrai, que la valeur de résistance d'une histoire autre de la vérité émerge avec le plus de force. Son personnage du cynique est l'un des exemples les plus intéressants de combat critique pour le lecteur foucaldien; un exemple difficile aussi, parce qu'il est compliqué à traduire dans une pratique politique, éthique, existentielle - d'autant qu'aucun d'entre nous ne choisira vraisemblablement de vivre dans la rue, mendier, revêtir des haillons, faire l'amour sur la place publique...

Foucault trace pourtant une ligne de questionnement précise liant la vérité, le soi et l'exercice critique, qui demeure à mon sens essentielle pour repenser la tâche de la pensée critique aujourd'hui (du moins au sens foucaldien du terme). Et cette forme de pensée de la résistance réside précisément dans ce «bien peu de vérité » qui est nécessaire à qui veut vivre vraiment. Vivre selon une vérité qui n'est pas à respecter, aimer, imiter, mais à détruire et toujours (re)construire, c'est la forme première de la liberté. La question du direvrai se brouille donc, là où « dire c'est faire »-ou « faire c'est dire »-, pour paraphraser Austin. Lorsque la vérité devient virtualité d'insurrection physique, elle perd son identité de vérité pour devenir élément de subversion des signes, vérités, discours, pratiques assujettissantes. La vraie postérité du cynisme et du geste foucaldien aujourd'hui serait alors à chercher moins du côté de la philosophie, dont l'académisme n'a fait que prospérer depuis la mort de Foucault, mais du côté des performances politico-esthétiques : des arts performatifs, des identités « véritablement faussées », des pratiques queer.

Il est intéressant pour cela de revenir sur la triade conceptuelle performatif-performance-dramatique. Comme on le sait, Foucault au début du cours au Collège de France de 1983, Le gouvernement de soi et des autres $^{22}$, définit son entreprise conceptuelle comme une analyse des structures «dramatiques » du discours politique, et il esquisse clairement les traits essentiels de cette dramatique des discours en la différenciant de la notion du performatif linguistique. La dramatique n'est ni une rhétorique ni une pragmatique du discours. Le vrai qui y est énoncé ne dépend ni de l'habilité verbale ni de la position sociale du locuteur. Il s'agit bien de «faire » avec les mots, comme dans l'énoncé performatif que la philosophie analytique anglo-saxonne avait analysé à partir des années soixante ${ }^{23}$. Mais Foucault retrace au moins trois différences fondamentales entre dramatique et performativité, dramatique et pragmatique des discours. D'abord, un énoncé performatif n'accomplit un acte que dans un cadre parfaitement établi, antérieur à l'énoncé lui-même. Le prêtre qui dit : «Je te baptise » en est un exemple : l'effet de cette formule rituelle dépend du cadre institutionnalisé du sacrement dans laquelle elle est prononcée. Le statut du sujet de l'énonciation est fondamental - il faut que le prêtre prononce ces mots en tant que ministre d'un sacrement, précisément. Mais ce n'est pas nécessaire que le sujet s'engage dans ce qu'il affirme. Que le prêtre soit ou non un bon chrétien, qu'il croie ou non en Dieu, cela ne conditionne en rien la validité des formules baptismales. Le baptême reste valide même en présence d'un ministre indigne.

Une vérité dramatique comme la parrêsia est quelque chose de très différent. Elle peut être dite par un sujet quelconque, sans rapport avec son statut, pourvu qu'il ait construit en soi les conditions éthiques pour l'affirmation de la vérité (pourvu qu'il accepte de se lier, de mettre en jeu soi-même et sa propre vie dans la vérité qu'il affirme). La position sociale ne compromet pas le dire-vrai, elle en fait au contraire un acte de courage quand il s'agit de s'adresser à quelqu'un de rang plus élevé, plus puissant que soi. En conséquence la vérité dramatique ne dépend pas d'un contexte institutionnel mais agit comme un vecteur de bouleversement du cadre socio-politique dans lequel elle s'affirme. Enfin, elle exige un lien solide de cohérence entre sujet et contenu de l'énonciation. Il faut que le locuteur affirme ce qu'il croit être vrai, qu'il devienne, au sens le plus fort du terme, sujet de vérité. À travers le projet d'une étude des différentes formes dramatiques de la vérité, il est donc clair que Foucault veut réfléchir sur une vérité qui ne soit ni une représentation du réel ni un pur rite mais une force de changement de soi-même et des autres. Une pratique plutôt qu'une théorie, un style d'existence plutôt qu'un ensemble de critères formels, une dynamique de résistance plutôt que la formulation des normes qui légitiment la véridiction. Une vérité théâtrale, pourraiton dire, en contraposition aux Idées éternelles de la vérité-adéquation. À la limite, une pratique de transformation où les dimensions même de la vérité et du langage, comme on l'a vu, se troublent.

\footnotetext{
${ }^{21}$ Ibid., p. 175.

${ }^{22}$ Cf. Foucault M., Le gouvernement de soi et des autres. Cours au Collège de France. 1982-1983, F. Gros éd., Paris, Seuil/Gallimard, 2008, p. 59-67.

${ }^{23}$ Cf. en particulier Austin J.L., Quand dire, c'est faire [1962], trad. G. Lane, Paris, Le Seuil, 1970 ; SEARLE J., Les actes de langage [1969], trad. H. Pauchard, Paris, Hermann, 1972.
} 
Si Foucault marque alors la distance entre l'énoncé performatif décrit par Austin et la parrêsia comme dire-vrai dramatique, il me semble évident d'un autre côté qu'il y a un lien fort entre les dramatiques de la vérité et la dimension contemporaine de la performance ${ }^{24}$. Il suit la même voie qui distingue le performatif artistique et théâtral du performatif linguistique : le discours dramatique de vérité retracé par Foucault et l'esthétique du performatif ne se fondent pas sur des statuts individuels prédéterminés mais créent de nouvelles formes de subjectivité ; ils ne peuvent pas être analysés avec des définitions préalablement construites du vrai et du faux, de l'efficace et de l'inefficace ; ils construisent la réalité, oui, mais d'une manière ouverte et imprévisible. On pourrait se risquer à dire que l'exigence critique qui traverse diagonalement la démarche foucaldienne pose à la philosophie les mêmes défis que la performance pose à l'art : elle indique une pratique de vérité qui ne vise pas à faire comprendre ou apprendre, mais à faire agir et réagir, dans un mouvement de déplacement et d'invention de nouvelles règles dans les « jeux » du vrai et du faux, dans les partages entre la nature et l'artifice, dans les impositions et les batailles des corps.

Il est significatif que l'une des réactivations contemporaines les plus intéressantes de la portée critique des généalogies foucaldiennes - la philosophie de Judith Butler - reprenne précisément la notion opératrice du performatif dans ce sens non-linguistique de performance, et dans un sens qui est très proche de la dimension dramatique de la pensée de Foucault. La dimension performatrice (notamment à propos du genre) est une répétition signifiante d'actions et la construction identitaire qui en résulte se retrouve à la surface des corps ; c'est « une construction de signification dramatique et contingente $»^{25}$, selon les mots de Butler ellemême dans son ouvrage Gender Trouble. Pour conclure, et sans prétention d'exhaustivité dans la restitution de la pensée butlerienne, il nous semble important de souligner que ses analyses des sujets queer et en particulier des drag queens comme acteur.e.s d'une subversion parodique des identités de genre sont une actualisation intéressante des sujets cyniques décrits par Foucault comme théâtre corporel et déformant du vrai, performance scandaleuse des conventions acquises. On retrouve la même valeur trouble d'un «activisme théâtral» (theatrical activism ${ }^{26}$, l'expression est de Butler) comme pratique politique de subversion, aux limites de la discursivité et de sa valeur de vérité.

\footnotetext{
${ }^{24}$ Je reprends ici des éléments des réflexions esquissées dans mon livre: Scènes de la vérité. Michel Foucault et le théâtre, Lormont, Le Bord de l'eau, 2017.

${ }^{25}$ BUTLER J., Gender trouble. Feminism and the subversion of identity, New York, Routledge, Routledge classics, 2006, p. 190 ; je traduis.

${ }^{26}$ BUTLER J., Bodies that matter. On the discursive limits of « sex », New York, Routledge, 1993, p. 233.
} 